\title{
Presenile dementia with motor neuron disease in Japan: clinico-pathological review of 26 cases
}

\author{
YOSHIO MITSUYAMA \\ From the Department of Psychiatry, Miyazaki Medical College, Miyazaki, Japan
}

SUMMARY The clinico-pathological findings of 26 cases of presenile dementia with motor neuron disease in Japan are reviewed. The characteristic features include: (1) Progressive dementia with slowly progressive onset in the presenile period. (2) Neurogenic muscular wasting during the course of illness. (3) A duration of illness to death of from one to three years. (4) Absence of extrapyramidal symptoms and definite sensory deficits. (5) No characteristic abnormalities in the CSF or EEG. (6) No known parental consanguinity of familial occurrence. (7) Non-specific mild degenerative changes throughout the CNS without evidence of cerebrovascular disease or primary degenerative dementia, but with the presence of pathological findings of motor neuron disease. The possibility that this is a new disease entity is suggested.

The author has previously reported several cases of presenile dementia with motor neuron disease, and has suggested that it is a new disease entity. ${ }^{1-3}$ This report describes a further clinico-pathological analysis of 26 cases in Japan, and defines the characteristic features of the disease.

Three typical cases examined by the author will be described briefly.

Case 1:1 A 60-year-old housewife at the age of 58 years developed an illness of which the initial symptom was memory difficulty. There was no previous personal or family history of neurological disease or psychosis. She was unaware of her disease and showed "Denkfaulheit" and "stenhende Redensarten" which are frequently seen in Pick's disease. She later developed anarthria. About one year after the onset of her illness, muscular atrophy in the arms, thenar, hypothenar, shoulder and bulbar muscles, with fasciculation, was observed. Deep tendon reflexes in the limbs were normal with no pathological reflexes. There was no rigidity or tremor. No myoclonus or convulsions occurred. Pneumoencephalography revealed diffuse cerebral atrophy, and EMG showed lower motor neuron damage. The EEG was regarded as normal. Routine laboratory studies and cerebrospinal fluid were within the normal range. The illness was progressive and she died about two years after the onset of the disease.

General pathology was unremarkable, except for acute bronchopneumonia as the immediate cause of death. The brain weighed $1,070 \mathrm{~g}$ and showed diffuse cerebral atrophy

Address for reprint requests: Yoshio Mitsuyama, MD, Department of Psychiatry, Miyazaki Medical College, Miyazaki 889-16 Japan

Received 22 February 1983 and in revised form 23 March 1984. Accepted 24 March 1984

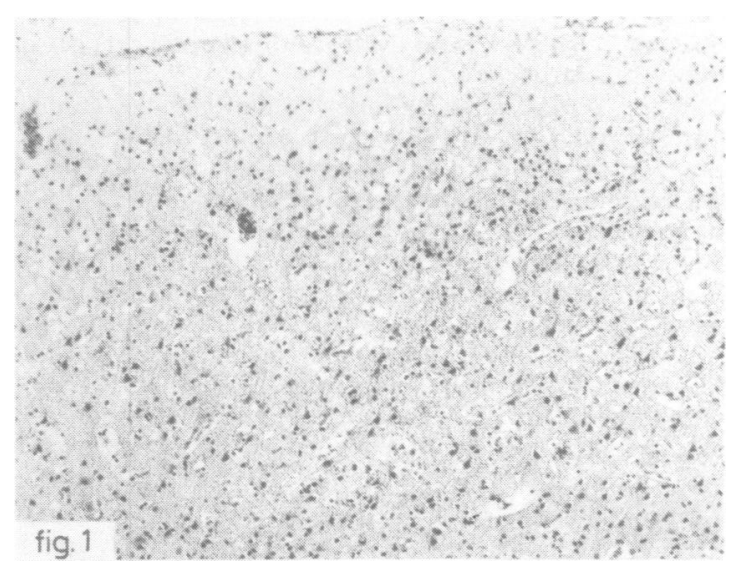

Fig 1 Case 1; Spongiose state in the superior frontal gyrus, $H-E$. stain $(\times 74)$.

which was marked in the frontal lobes. Microscopic findings revealed that the cerebral cortex in all areas exhibited a moderate degree of nerve cell degneration, characterised by simple atrophy and pigmentary sclerosis, and some loss of neruons with a astroglial reaction. There was a mild spongiose state between the second and third layers in the frontal, temporal and central cortices (fig. 1). The deep white substance of the cerebral hemisphere revealed only a mild rarefaction of myelin sheaths with mild astrogliosis. In the lateral tract of the spinal cord, there was no evidence of demyelination. In the medulla oblongata, reduction of hypoglossal nuclei was evident and there was marked loss of anterior horn cells in the cervical and thoracic cords (fig. 2). Muscles from the upper extremity showed neurogenic atrophy (fig. 3). No definite lesions in striatum, 


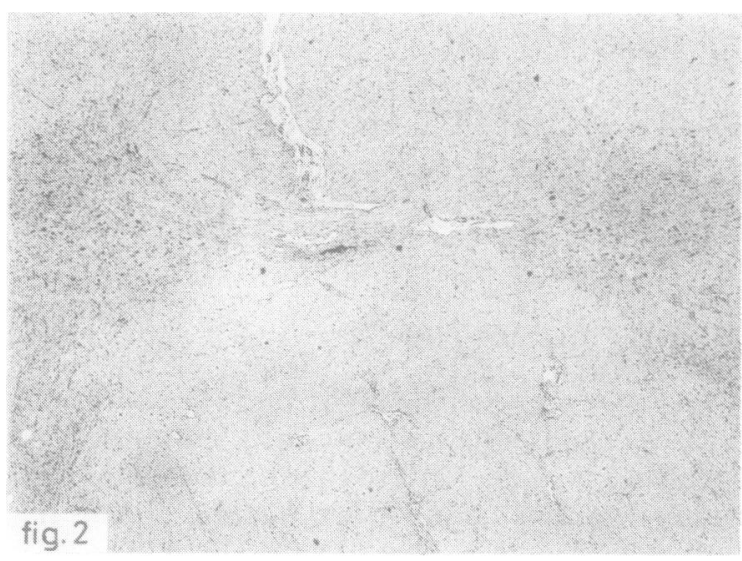

Fig 2 Case 1; Loss of anterior horn cells in the cervical cord, Nissl stain $(\times 15)$.

thalamus and substantia nigra was demonstrated. There were many torpedos in the cerebellum. Using the Bodian silver impregnation method, no senile plaques, Alzheimer's neurofibrillary tangles or granulovacuolar degeneration was observed in any area, including the hippocampus. There was no evidence of sclerotic changes of the vessels throughout CNS.

Case 2:2 A 61-year-old housewife suffered the gradual onset of difficulty with memory, concentration and cognition at the age of 58 years. Progressively, severe dementia was accompanied by muscle wasting and fasciculation prominent in the hand and bulbar muscles (fig. 4). Behavioral changes and mental retardation became prominent. Progressive worsening of her speech and imperfect use of the upper extremities developed. Muscle strength was moderately to severely diminished in the arms and to a lesser extent in the legs, most prominent distally. The muscles of the thighs and legs were not atrophied. The muscle stretch reflexes were normal in the arms but hyperactive in

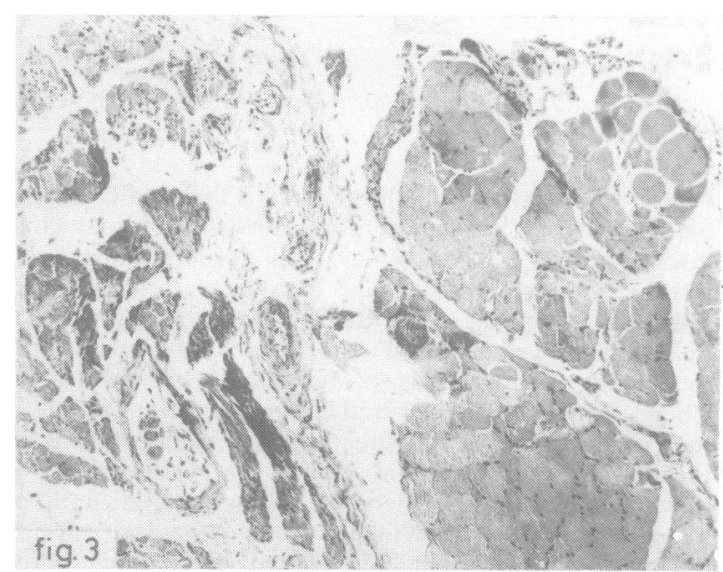

Fig 3 Case 1; Neurogenic muscle atrophy in hand muscle, H-E. stain $(\times 74)$.

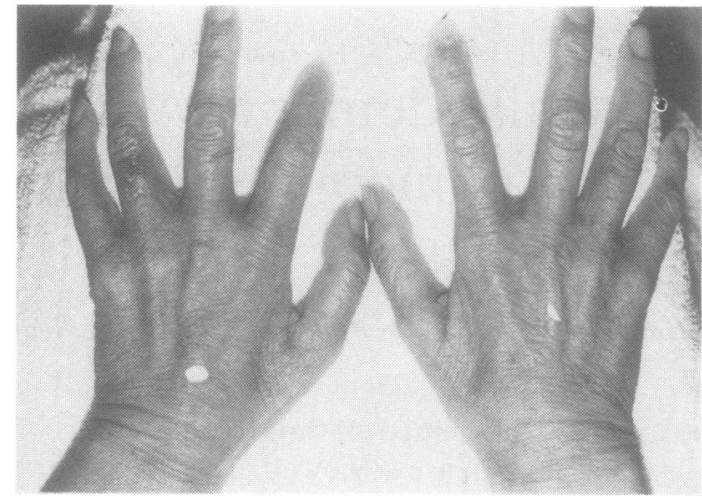

Fig 4 Case 2; Atrophy in hand muscle.

the legs. There was no Babinski's sign. The Romberg test was negative. There was no ataxia, rigidity or tremor. No myoclonic jerks or convulsions were observed. Routine laboratory studies and cerebrospinal fluid were normal. A CT scan of the brain disclosed considerable cerebral atrophy, especially in the fronto-temporal regions, with considerable enlargement of the ventricular system. An EEG demonstrated slow background activity with frequent theta activity especially in the fronto-temporal leads. There were no high voltage periodic synchronous spikes, spike and wave rhythm, or triphasic complexes. A biopsy specimen from the right biceps was interpreted as showing $\propto$ neurogenic atrophy. An EMG showed frequent fibrilla- $\overline{0} \mathbb{D}$ tions and fasciculations. There were some polyphasic $\cap$ motor units. Nerve conduction velocities were normal. The음 patient died two years and six months after the onset of her illness and permission for necropsy was not obtained.

Case $3:^{3}$ A 53-year-old male was hospitalised because of progressive dementia and muscular weakness of the upper extemities, which had begun one year previously. There was no history of preceding febrile illness, neurological disease or psychosis, and no relative with a similar disease. The patient had worked as a construction labourer for $\mathbf{3 0}$ years. At age 50 years, he developed progressive difficulty in remembering recent events and in concentration, with an inability to perform his usual occupation. Several months before admission, his speech became dysarthric and he had difficulty swallowing. Behavioral changes and mental deterioration became prominent. His family noticed progressive worsening of his speech. Finally he became so emaciated and in such a state of infirmity that he was hospitalised.

On admission, he was alert and orientated as to time, person and place. He answered questions slowly. His calculations were performed poorly; writing and spelling were also poor. Although he walked steadily, he appeared apathetic, had difficulty in understanding and spoke only a few words. He had severe dysphasia and was unaware of his disease. Memory, attention and cognition were severely impaired. Psychometric tests could not be performed because of his poor comprehension. An examination of the cranial nerves showed involvement of the IX, X, XI and XII nerves. There was generalised weakness and the wast- 


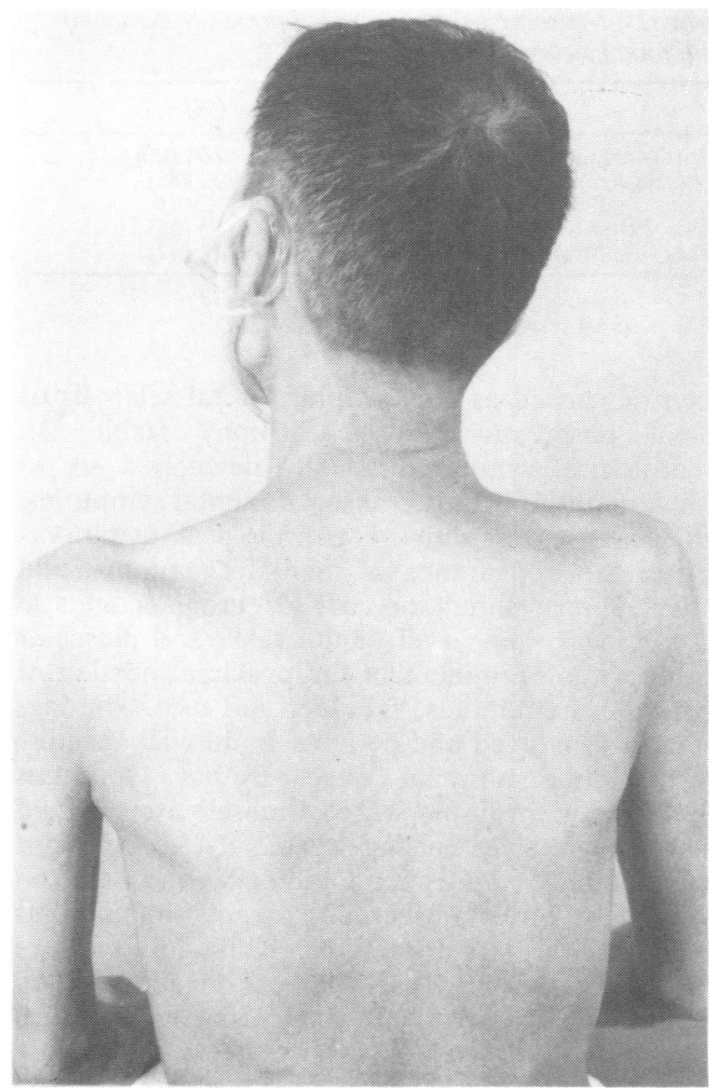

Fig 5 Case 3; Muscle atrophy in shoulders.

ing of musculature in the extremities, more prominent in the arms and shoulders, and in the bulbar muscles (fig. 5). There was marked dysarthria and difficulty in swallowing. His tongue was slightly atrophic with fasciculations and its movements were weak. Palatal and pharyngeal reflexes were decreased. Muscle strength was moderately induced in the arms and diminished to a lesser degree in the lower legs, most prominent proximally. The muscles of the thighs and legs were atrophic. Marked fasciculation was seen in the arms. He was able to walk without support. The muscle stretch reflexes were symmetrically hyperactive in all extremities with bilateral extensor plantar responses. Snout, glabellar, and palmo-mental reflexes were not present. Sensation could not be tested because of his inability to cooperate. Abdominal reflexes were present and symmetrical. The Romberg test was negative. There was no rigidity or tremor. No myoclonus or convulsions occurred. Routine laboratory studies and cerebrospinal fluid examined by repeated lumbar punctures disclosed no abnormality. A CT scan of the brain revealed slight cerebral atrophy, mostly concentrated in the fronto-temporal regions with considerable enlargement of the ventricular system without shift (fig. 6). An EEG, 10 months after the onset of symptoms, showed a slight slowing of the back-

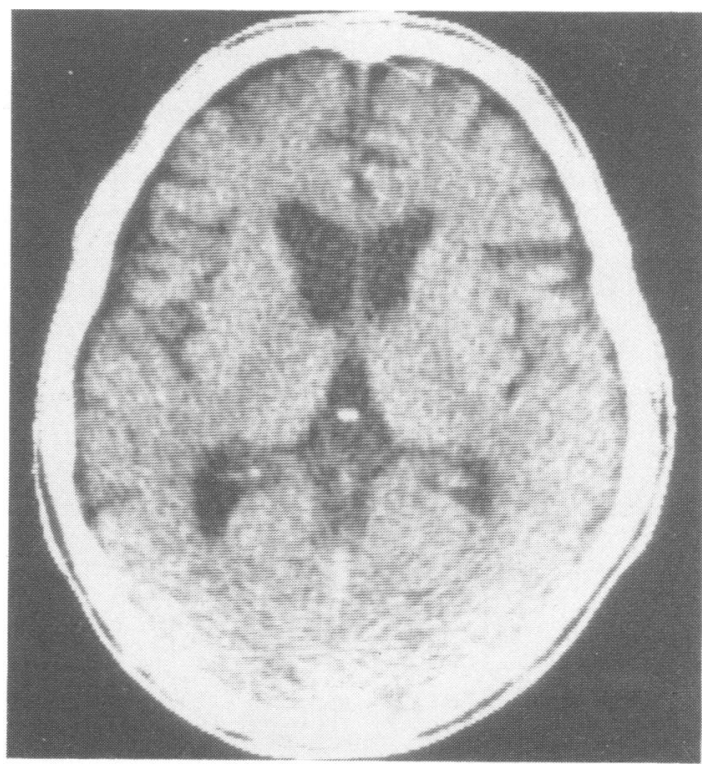

Fig 6 Case 3; CT scan shows moderate cerebral atrophy.

ground activity at 8 to $10 \mathrm{~Hz}$., intermixed with occasional theta over both hemispheres. There were no periodic, triphasic sharp waves. Muscle specimen from the right deltoid was interpreted as showing neurogenic atrophy (fig. 7). The EMG revealed frequent fibrillation and fasciculation; motor units showed polyphasia, and nerve conduction vectors were normal. His condition continued to deteriorate and he died two years and nine months after the onset of his illness. Permission for necropsy was not obtained.

Twenty six similar cases of presenile dementia with motor neuron disease have been reported in Japan between 1964 and 1983.There is no sex difference in the incidence (female: 13, male: 13), age at onset (female: $38-68$ years, average: $54 \cdot 1$; male: 43-68, ave.: 52.4) and death (female: 39-69 years, ave.: $57 \cdot 7$, male: $43-68$, ave.: $54 \cdot 8$ ). The duration of illness of both sexes is from one to six years (ave.: range 2.5 years). No localised area of incidence in Japan has been observed. Patients showed progressive dementia with insidious onset in the presenile period. The main mental symptoms are shown in table 1 . They consisted of memory difficulty, global intellectual impairment, personality change, emotional disorders and loss of spontaneous speech. These symptoms were usually interpreted as unclassified presenile dementia. They were not typical of Alzheimer or Pick disease in their clinical course. Reduction of spontaneous speech seemed to be more characteristic of the early stage of the illness and patients usually did not develop into a severely deteriorated state even in an advanced 


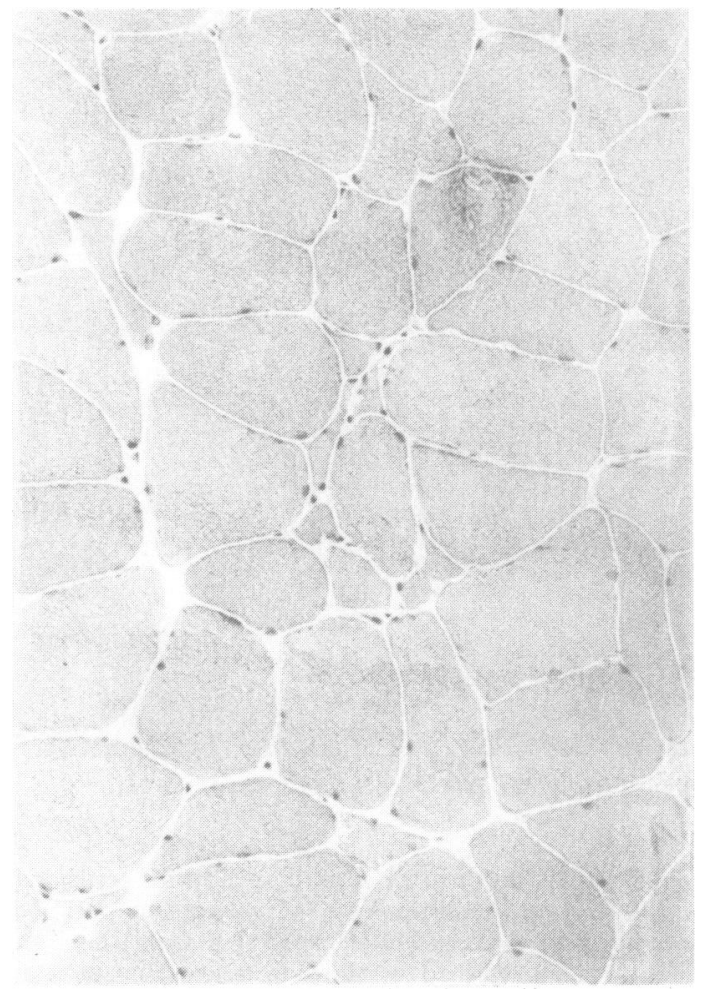

Fig 7 Case 3; Muscle biopsy from the right deltoid shows neurogenic atrophy. $(H-E . \times 200)$.

stage of the disease. Personality change also was mild to slight and personal contact preserved fairly well. There was great difference between Pick disease and the mental symptoms of presenile dementia with motor neuron disease. No apraxia or agnosia, which frequently are seen in Alzheimer disease, were found in any case.

Neurological symptoms of presenile dementia with motor neuron disease include: neurogenic muscle atrophy, bulbar symptoms (dysphagia, dysarthria), and occasional pyramidal signs. Patients have

Table 1 Mental symptoms of presenile dementia with MND $(n=26)$

\begin{tabular}{ll}
\hline & $(\%)$ \\
\hline Memory impairment & $26(100)$ \\
Global intellectual impairment & $25(96)$ \\
$\begin{array}{l}\text { Personality change } \\
\text { Emotional disorders }\end{array}$ & $22(85)$ \\
$\quad$ (euphoria, indifference, incontinence) & $22(85)$ \\
$\begin{array}{l}\text { Loss of speech, perseveration, } \\
\text { stereotyped speech }\end{array}$ & $15(58)$ \\
Pick-like symptoms & $8(31)$ \\
Alzheimer-like symptoms & $4(15)$ \\
Unclassified & $14(54)$ \\
\hline
\end{tabular}

Table 2 Neurological symptoms of presenile dementia with motor neuron disease in Japan

\begin{tabular}{ll}
\hline & $(\%)$ \\
\hline Neurogenic muscle atrophy & $26(100)$ \\
Dysarthria, dysphagia & $22(85)$ \\
Babinski refiex & $2(8)$ \\
Cases with ALS-like symptoms & $15(58)$ \\
Cases with SPM-like symptoms & $11(42)$ \\
\hline
\end{tabular}

ALS = amyotrophic lateral sclerosis

SPM = spinal progressive muscular atrophy

been diagnosed as amyotrophic lateral sclerosis or spinal progressive muscle atrophy (table 2). Neurological symptoms usually developed six to twelve months after the onset of mental symptoms, but some cases showed neurological symptoms before the appearance of mental symptoms, and others almost simultaneously. Neurogenic muscle atrophy was seen in all of the cases and muscular wasting was prominent in the proximal portion of the upper extremities, shoulder and face. The legs were less involved and patients could walk steadily even in the terminal stage. Bulbar symptoms became evident in the stage of muscle atrophy and were progressive causing death. Pyramidal signs such as increased deep tendon reflexes and Babinski signs were rarely observed. Extrapyramidal signs such as rigidity, tremor and myoclonic jerking were not observed in any case. Patients did not develop generalised rigidity or spasticity in their clinical course.

Eighteen of the 26 cases of presenile dementia with motor neuron disease which were reviewed had pathological studies of brain and spinal cord. General pathology showed bronchopneumonia as an immediate cause of death in all cases. Gross inspection of the brain in the majority of these cases showed atrophy that varied from slight to moderate, and was mainly in the frontal and/or temporal lobes. Principal neuropathological findings are shown in table 3. Non-specific neuronal degeneration, characterised by simple atrophy and pigmentary sclerosis in the cerebral cortex, coexisted with lesions similar to those seen in motor neuron disease such as

Table 3 Review on pathology $(n=18)$

\begin{tabular}{ll}
\hline & $(\%)$ \\
\hline $\begin{array}{l}\text { Neuronal loss and degeneration in } \\
\text { cerebral cortex. }\end{array}$ & $18(100)$ \\
Neurogenic muscle atrophy & $18(100)$ \\
Neuronal loss of anterior horn cells & $16(89)$ \\
$\quad$ in spinal cord. & $16(89)$ \\
Loss of hypoglossal nuclei. & $14(78)$ \\
Subcortical gliosis of cerebrum. & $9(50)$ \\
Cortical spinal tract degeneration. & $4(22)$ \\
Sponginess in cerebral cortex. & $4(22)$ \\
Senility related changes. &
\end{tabular}


neurogenic muscle atrophy; lesions in the spinal cord and hypoglossal nuclei were more commonly found in the case of presenile dementia with motor neuron disease. Lower motor neuron degeneration was observed in 16 cases. There were degenerative changes with simple atrophy and pigmentary sclerosis and a mild to slight disappearance of neurons in the cerebral cortex in all cases and these changes were predominent in layers 2 and 3 in the frontal and temporal regions. Fibrillary astrogliosis in the subcortical white substance of the cerebral hemisphere was frequently observed. Occasionally, a mild spongy appearance of the cortex was present in the 2 nd and third layers. Using the silver impregnation method, senile plaques, Alzheimer's neurofibrillary tangles and granulovacuolar degeneration were found infrequently and they were localised in the hippocampus. Although half of the subjects showed pyramidal tract degeneration, it was very mild compared with that seen in the classical sporadic amyotrophic lateral sclerosis. Degeneration of substantia nigra or globus pallidus was frequently reported and neuronal loss or gliosis was found in other neuronal structures, such as the corpus striatum, thalamus and corpus Luysii, in some cases. There was no evidence of demylination of the cerebral white substance in any case. There was no case with cerebrovascular lesions caused by arteriosclerotic vessel disease.

\section{Discussion}

Presenile dementia with motor neuron disease was first described by Yuasa ${ }^{4}$ in Japan. There are 26 case reports of presenile dementia with motor neuron disease which, for the most part, satisfy the clinical criteria described by the author. ${ }^{2}$ Eighteen of them have had pathological examinations of the central nervous system. An epidemiological review of presenile dementia with motor neuron disease has shown an approximately equal male to female ratio, mean age of onset 53 years, and mean age at death 56 years, which are almost similar to those with classical sporadic amyotrophic lateral sclerosis. All patients have had prominent progressive dementia and development of motor neuron disease. The dementia in these cases had, in the main, the same clinical features as occur in other known primary degenerative dementia such as Alzheimer disease, Pick disease, progressive subcortical gliosis ${ }^{5}$ and Creutzfeldt-Jakob disease. However, selective cognitive dysfunction, language impairment such as logoclonia, and visuo-spatial disorientation that are frequently seen in Alzheimer disease were not observed in any case of presenile dementia with motor neuron disease. "Stehende Redensarten" that is frequently observed in Pick disease was seen in several cases. A CT scan of the head in all these cases showed fronto-temporal atrophy and mental symptoms were suggestive of Pick disease rather than Alzheimer disease. Although motor neuron disease appeared before or at the same time as organic dementia in some cases, neurological symptoms appeared usually six to twelve months after the onset of the mental symptoms.

Classical amyotrophic lateral sclerosis is described as a disease of progressive wasting and weakness of the bulbar and spinal skeletal musculature with variable signs of fasciculation and pyramidal impairment due to degeneration of the lower and upper motor neurons, In the cases described here, there were usually no definite pyramidal signs or pathological reflexes and the distribution pattern of muscle atrophy was not identical to that found in classical amyotrophic lateral sclerosis. Although there are a number of reports of demenita, Parkinsonism and sensory changes occurring in association with amyotrophic lateral sclerosis, ${ }^{614}$ advanced dementia has not been reported in classical amyotrophic lateral sclerosis, and amyotrophic lateral sclerosis-like symptoms usually have not been described in classical Alzheimer disease or Pick disease. Muscular weakeness and wasting have been described early in the course of Pick disease and during the terminal phase of Alzheimer disease. ${ }^{15}$ Lower motor neuron and pyramidal tract degeneration occur as part of widespread abnormalities of the nervous system in Creutzfeldt-Jakob disease. None of these cases resembled the classical form of Creutzfeldt-Jakob disease, but the amyotrophic form of CreutzfeldtJakob disease is clinically indistinguishable from these cases of presenile dementia with motor neuron disease. The cases described here may belong to this amyotrophic form because of a long clinical course with mental deterioration and amyotrophy.

The pathological findings in these cases of presenile dementia with motor neuron disease were essentially similar. All the necropsies of presenile dementia with motor neuron disease showed evidence of diffuse degenerative changes in the cerebral hemisphere and spinal cord. Cerebral cortical atrophy was present in every case of presenile dementia with motor neuron disease and this was mainly fronto-temporal. Neuronal loss and degeneration characterised by simple atrophy and pigmentary sclerosis and mild status spongiosus in the upper layer were the main pathological features in the cortex. They were not consistent with those of Alzheimer disease or Pick disease, and clinicopathological information allowed no other definitive diagnosis. Fronto-temporal regions were predominently involved. Fibrillary astrogliosis in subcortical 
white substance was observed with variable severity from case to case as a normal finding by the author or more pathological in the case of other authors. Pyramidal tract degenerative change was not common in the cases of presenile dementia with motor neuron disease. Clinically and histopathologically, we see some resemblance to Creutzfeldt-Jakob disease, amyotrophic lateral sclerosis and progressive subcortical gliosis. The cases described here generally revealed mild histopathological changes, and there was no evidence of definite spongy change with astrogliosis in the cerebral cortex to support the diagnosis of Creutzfeldt-Jakob disease. Lesions in the brain stem and spinal cord were very different from those in progressive subcortical gliosis.

A differential diagnosis between these cases and amyotrophic lateral sclerosis with dementia and/or Parkinsonism as is seen in Guam ${ }^{16}$ should be discussed. It has been reported that the presence of Alzheimer's neurofibrillary tangles in the hippocampus and substantia nigra is a paramount finding in Guamanian amyotrophic lateral sclerosis and Parkinsonism-dementia complex in Guam. ${ }^{16}{ }^{17}$ There is no case report with neuropathological findings compatible with Guamanian amyotrophic lateral sclerosis or Parkinsonism-dementia complex in presenile dementia with motor neuron disease. Similar cases have been reported in other countries $^{18-23}$ and they have been regarded as subtypes of amyotrophic lateral sclerosis, Creutzfeldt-Jakob disease, Alzheimer disease or Pick disease. We see some different characteristics in these cases described here and cannot make a diagnosis of amyotrophic lateral sclerosis or Pick disease from the clinico-pathological findings. Although Hudson $^{10}$ and Salazar et al. ${ }^{24}$ observed that dementia is part of amyotrophic lateral sclerosis syndrome from the review of amyotrophic lateral sclerosis-dementia case reports, pathological studies of the nervous system in presenile dementia with motor neuron disease showed a low incidence of pyramidal tract degeneration. This was not similar to classical sporadic amyotrophic lateral sclerosis. It is proposed that primary degenerative dementia is a principal disorder and motor neuron disease is part of the same process from their clinico-pathological studies. The similarity of their clinical manifestations and pathological findings in the necropsies suggests that presenile dementia with motor neuron disease may be a single disease rather than a variant of amyotrophic lateral sclerosis or Creutzfeldt-Jakob disease, but these findings need to be confirmed in a large series of cases. Although transmissibility has not been determined in the case of the amyotrophic form of Creutzfeldt-Jakob disease, ${ }^{25}$ the experimental transmission to animals is recommended.

\section{References}

${ }^{1}$ Mitsuyama Y, Takamatsu I. An autopsy case of presenile dementia with motor neuron disease. Brain, Nerve (Tokyo) 1971;23:409-16.

${ }^{2}$ Mitsuyama Y, Takamiya S. Presenile dementia with motor neuron disease. Arch Neurol 1979;36:592-3.

${ }^{3}$ Mitsuyama Y, Tobo M. Presenile dementia with motor neuron disease. An additional case report. Folia Psychiat Neurol Jpn 1981;35:35-42.

${ }^{4}$ Yuasa R. Amyotrophic lateral sclerosis with organic dementia: Report of a case. Clin Neurol (Tokyo) 1964;4:529-34.

5 Neumann MA, Cohen R. Progressive subcortical gliosis. A rare form of presenile dementia. Brain 1967; 90:405-18.

- Brait K, Fahn S, Schwarz GA. Sporadic and familial parkinsonism and motor neuron disease. Neurolgy (Minneap) 1973;23:990-1002.

${ }^{7}$ Brownell B, Oppenheimer DR, Hughes JT. The central nervous system in motor neuron disease. $J$ Neurol Neurosurg Psychiatry 1970;33:338-57.

${ }^{8}$ Finlayson MH, Guberman A, Martin JB. Cerebral lesions in familial amyotrophic lateral sclerosis and dementia. Acta Neuropathol 1973;26:237-46.

9 Hakin AM, Mathieson G. Dementia in parkinson disease. A neuropathologic study. Neurology (Minneap) 1979; 29: 1209-14.

${ }^{10}$ Hudson AJ. Amyotrophic lateral sclerosis and its association with dementia, parkinsonism and other neurological disorders: a review. Brain $1981 ; 104: 217-48$.

"Ishii N, Nishimaru K, Okumura M, Kikuchi M. A case of bulbar palsy, parkinsonism and dementia with diffuse neurofibrillary changes. Clin Neurol (Tokyo) 1974;16:483-90.

12 Jokelainen M. Amyotrophic lateral sclerosis in Finland. II. Clinical characteristics. Acta Neurol Scand 1977; 56: 194-204.

${ }^{13}$ Kaiya $\mathrm{H}$, Mehraein P. Clinical and pathological findings in the syndrome of amyotrophy-parkinsonismdementia. Arch Psychiat Nervenkrh 1974;219:13-27.

${ }^{14}$ Smith MC. Nerve fibre degeneration in the brain in amyotrophic lateral sclerosis. J Neurol Neurosurg Psychiatry 1960;23:269-82.

is Balajthy B. Symptomatology of the temporal lobe in Pick's convolutional atrophy. Acta Med Acad Soc Hung 1964;20:304-16.

${ }^{16}$ Hirano A, Malamud E, Elizan TS, Kurland LT. Amyotrophic lateral sclerosis and parkisonism-dementia complex on Guam. Further pathologic studies. Arch Neurol 1966;15:35-51.

17 Hirano A, Zimmerman HM. Alzheimer's neurofibrillary changes. A topographic study. Arch Neurol 1962; 7:227-42.

${ }^{18}$ Minauf $M$, Jellinger $K$. Kombination von amyotrophischer Lateralsklersoe mit Pickscher Krankheit. Arch Psychiat Nervenkr 1969;212:279-88.

${ }^{19}$ Myrianthopoulos NC, Smith JK. Amyotrophic lateral sclerosis with progressive dementia. Neurology (Minneap) $1962 ; 12: 603-10$.

${ }^{20}$ Poppe W, Tennstedt A. Klinisch- und pathologisch- 
anatomische Untersuchungen ueber Kombinationsformen praeseniler Hirnatrophien (Pick, Alzheimer) mit spinalen atrophisierenden Prozessen. Psychia Neurol 1963;145:322-44.

${ }^{21}$ von Braunmuehl A. Picksche Krankheit und amyotrophische Lateralsklerose. Allg Z Psychiat 1932;96:364-6.

22 von Matt K. Progressive Bulbaerparalyse und dementielles Syndrome. Psychiat Neurol 1964;148:354-64.

${ }^{23}$ Wikstroem J, Paetau A, Palo J, Sulkava R, Haltia M.
Classic amyotrophic lateral sclerosis with dementia. Arch Neurol 1982;39:681-3.

${ }^{24}$ Salazar AM, Masters CL, Gajdusek DC, Gibbs CJ. Syndromes of amyotrophic lateral sclerosis and dementia: Relation to transmissible Creutzfeldt-Jakob disease. Ann Neurol 1983;14:17-26.

${ }^{25}$ von Rossum A. Spastic pseudosclersosis (CreutzfeldtJakob disease). In; Handbook of Clinical Neurology. Disease of the Basal Ganglia, Vinken PJ, Gruyn GW, eds New York, Elsevier, vol. 6, 1968;726-60. 